\title{
Human papillomavirus type 18 oncoproteins exert their oncogenicity in esophageal and tongue squamous cell carcinoma cell lines distinctly
}

Siaw Shi Boon ${ }^{1 \dagger}$, Zigui Chen ${ }^{1+}$, Jintao Li $^{2}$, Karen Y. C. Lee ${ }^{1}$, Liuyang Cai ${ }^{1}$, Rugang Zhong ${ }^{2}$ and Paul K. S. Chan ${ }^{1 *}$ (D)

\begin{abstract}
Background: Increasing evidence indicates an etiological role of human papillomavirus (HPV) in head and neck cancers, particularly oropharyngeal squamous cell carcinoma (OPSCC). However, the association between HPV and other cancers, including esophageal and tongue remains unclear. This study delineated the molecular characteristics of HPV18 E6 and E7 in esophageal (EC109 and EC9706) and tongue (Tca83) cancer cell lines with reference to cervical cancer (HeLa).

Methods: We analysed the HPV transcription profiles of esophageal and tongue cancer cells through Nextgeneration RNA sequencing, and the role of HPV18 E6 and E7 in these cells was assessed via siRNA approach, Western blotting and immunofluorescence assays.

Results: Overall, the HPV transcription profiles of esophageal and tongue cancer cells mimicked that of cervical cancer cells, with notable disruption of E2, and expression of E6, spliced E6 (E6 $\left.{ }^{*}\right), E 7, E 1$ and L1 transcripts. As with cervical cancer cells, p53 and its downstream transactivation target, p21, were found to be the major targets of E6 in esophageal and tongue cancer cell lines. Intriguingly, E7 preferentially targeted p130 in the two esophageal cancer cell lines, instead of pRb as in cervical cancer. Tca83 exhibited an E7 to E6 transcript ratio comparable to HeLa (cervix), targeted the ERK1/2 and MMP2 pathways, and was dependent on E6 and E7 to survive and proliferate. In contrast, both the esophageal cancer cell lines were distinct from HeLa in these aspects.
\end{abstract}

Conclusions: This is the first study that delineates transcript expression and protein interaction of HPV18 E6 and E7 in esophageal and tongue cancer cell lines, suggesting that HPV plays a role in inducing these cancers, albeit via distinct pathways than those observed in cervical cancer.

Keywords: HPV18, Esophageal squamous cell carcinoma, Tongue squamous cell carcinoma, E6, E7

\section{Background}

Head and neck cancers (HNC) and esophageal cancers (EC) are ranked the seventh and sixth most common causes of cancer death worldwide, respectively [1]. HNC incidence poses a worrisome increment in many geographical regions. It was estimated that the incidence of oropharyngeal cancers might further increase in the

\footnotetext{
* Correspondence: paulkschan@cuhk.edu.hk

+Siaw Shi Boon and Zigui Chen contributed equally to this work.

${ }^{1}$ Department of Microbiology, Faculty of Medicine, The Chinese University of Hong Kong, Shatin, Hong Kong

Full list of author information is available at the end of the article
}

United States and European countries [2-4]. Meanwhile, esophageal squamous cell carcinoma is highly prevalent in the so-called "esophageal cancer belt", including Northern Iran, Central Asia, North-Central China, along the Rift Valley in East Africa, and South Africa [5]. Among these countries, China is the most affected, particularly in rural areas such as the Henan province [5].

Both HNC and EC appear to share similar risk factors, including poverty, alcohol and tobacco consumption [6, 7], diet and nutrition $[8,9]$, as well as exposure to environmental carcinogens such as polycyclic aromatic hydrocarbons (PAHs) [10, 11]. Human papillomavirus (HPV), mainly

(c) The Author(s). 2019 Open Access This article is distributed under the terms of the Creative Commons Attribution 4.0 International License (http://creativecommons.org/licenses/by/4.0/) which permits unrestricted use, distribution, and reproduction in any medium, provided you give appropriate credit to the original author(s) and the source, provide a link to the Creative Commons license, and indicate if changes were made. The Creative Commons Public Domain Dedication waiver (http://creativecommons.org/publicdomain/zero/1.0/) applies to the data made available in this article, unless otherwise stated. 
HPV16 followed by HPV18, is now recognized as a cause of a fraction of oropharyngeal cancers [12-14]. However, the etiological role of HPV in tongue and esophageal cancers is still controversial [15-18]. HPV-induced carcinogenesis is mainly driven by the viral oncoproteins, E6 and E7, which are essential in maintaining tumor phenotype. E6 and E7 are multi-functional proteins involved in several cellular processes, including caspase-mediated apoptosis, cell cycle progression and signaling pathways. E6 mediates downregulation of p53 [19-21] and $\underline{\mathrm{PSD}}$ 95/lolg/ZO-1 (PDZ) proteins [22-24], leading to perturbed p21 functions and cell polarity, respectively. Whilst E7 downregulates retinoblastoma protein $(\mathrm{pRB})$ and its related pocket proteins, including p130 and p107 [25-27], leading to transactivation of E2F response promoter genes [28]. In addition, HPV oncoproteins can deregulate AKT [29], ERK [30] and metalloproteases (MMPs) [31, 32], leading to cancer formation and progression. All these are classical molecular targets of HPV oncoproteins in cervical cancer.

Observations at molecular level from established cancer cell lines could improve our understanding on the role of HPV in esophageal and tongue squamous cell carcinoma (SCC). In this study, we analyzed the transcript expression profiles and functions of E6 and E7 to delineate the role of HPV18 in esophageal (EC109 and EC9706) and tongue (Tca83) cancers based on cell lines established from Chinese.

\section{Methods}

\section{Cell lines}

Esophageal squamous cell carcinoma (SCC) (EC109 and EC9706) and tongue SCC (Tca83) cell lines were derived from patients in China. We included HeLa cells (HPV18-positive) originally derived from cervical cancer, and HKESC01 (HPV-null) from an esophageal cancer patient of Chinese origin, as references. HeLa cell line was purchased from the American Type Culture Collection (ATCC). The EC109, EC9706 and Tca83 cell lines were generous gifts from Prof. Zeng Yi, the National Institute for Viral Disease Control and Prevention of Chinese Center for Disease Control and Prevention in 2012. The HKESC01 cell line was a generous gift from Prof. Chi Hin Cho, the Chinese University of Hong Kong in 2017. All these cell lines have been authenticated using Short Tandem Repeat (STR) profiling this year. These mycoplasma-free cells were maintained in Dulbecco's Modified Eagle Medium (DMEM), supplemented with $10 \% \mathrm{FBS}$ in a $37^{\circ} \mathrm{C} \mathrm{hu-}$ midified incubator containing $5 \% \mathrm{CO}_{2}$.

\section{Next-generation RNA sequencing and bioinformatics analysis}

Total RNA of each cell line was extracted using RNeasy Mini Kit (Qiagen), treated with DNase, and prepared for
Next-generation sequencing library using TruSeq Stranded Total RNA LT (Illumina), according to the manufacturer's instructions. Briefly, libraries were run on an Illumina HiSeq4000 for paired-end $100 \mathrm{bp}$ sequencing. The RNAseq data were analyzed according to the HISAT2StringTie-Ballgown pipeline [33]. In brief, high-quality reads filtered by Trimmomatic V0.38 were mapped to the human genome index (GRCh38) using HISAT2 v2.1.0 with default parameters. A read coverage table was generated using StringTie v1.3.5 against a GRCh38 gtf annotation file, following the normalization procedure using Fragments per kilobase of transcript per million reads mapped (FPKM). We also built a HISAT2 transcript index and a gtf annotation file for HPV18 [34]. The R package Ballgown was used to create differential expression tables and plot gene transcript patterns. The RNA sequence data have been deposited in the NCBI Gene Expression Omnibus database (GEO, http://www.ncbi.nlm.nih.gov/geo/) and are accessible through GEO Series accession number (SRA Accession: PRJNA530677).

\section{Downregulation of HPV18 E6 and E7}

Approximately $2 \times 10^{5}$ of all the cells were seeded into 6-well plates. After 24h, HPV18 E6 and E7 expression were ablated by transfecting the cells with small interfering RNA (siRNA) against HPV18 E6/E7 (5'CAU UUA CCA GCC CGA CGA G) (siE6/E7) using Lipofectamine LTX reagent (Invitrogen) for $72 \mathrm{~h}$, according to protocol recommended by the manufacturer. Simultaneously, an independent set of cells were transfected with siControl (Dharmacon) to serve as a non-targeting siRNA negative control.

\section{Western blotting}

Total cell extracts were obtained by lysing the cells directly using $2 \times$ SDS-PAGE sample buffer. Western blotting and processing were then performed as described previously [35]. The following antibodies were used: mouse monoclonal anti-human pRB (BD Pharmingen); rabbit polyclonal anti-MMP2 and MMP9 (Abcam); rabbit monoclonal p21 (12D1), rabbit polyclonal phospho-Akt (Ser473), rabbit monoclonal pan Akt (Cell Signaling), mouse monoclonal anti-p53 (DO-1), mouse monoclonal anti- $\beta$-actin, mouse monoclonal anti-SAP97 (2D11) (Dlg), rabbit polyclonal p130 (C-20) and p107 (C-18), mouse monoclonal p-ERK1/ 2 (12D4), and ERK1/2 (C-9) were from Santa Cruz.

Immunoblots were developed using Clarity ${ }^{\mathrm{Tm}}$ Western ECL Substrate (Bio-Rad) and images were captured using the ChemiDoc ${ }^{\text {Tw }}$ Imaging System (Bio-Rad). Protein band intensities were quantified using ImageJ and normalized with the levels of $\beta$-actin, which serves as a loading control. 


\section{Immunofluorescence}

Approximately $2 \times 10^{5}$ cells were plated onto coverslips. After overnight incubation, cells were transfected with siRNA against HPV18 E6 and E7 (siE6/E7) or siControl, as described above. After $72 \mathrm{~h}$, cells were fixed with icecold absolute methanol. Cells were then incubated with specific primary antibodies against Ki67 (Santa Cruz) and p53 (Cell signaling), followed by relevant Alexa Fluor ${ }^{\circ} 568$-conjugated anti-rabbit and Alexa Fluor ${ }^{\circ} 488$ conjugated anti-mouse secondary antibodies (ThermoFisher Scientific), and counterstained with 4',6-diamidino-2-phenylindole (DAPI). Cells were examined under a fluorescence microscope (Leica).

\section{Data availability statement}

As mentioned above, the RNA sequence data is accessible through GEO Series accession number (SRA Accession: PRJNA530677). Data can be made available upon request.

\section{Results}

Esophageal and tongue cancer cell lines shared similar expression profile with cervical cancer cells, but exhibited different E7/E6 ratios

Viral genome integration resulting in disruption and loss of viral transcripts are remarkable features of HPVmediated oncogenesis. Therefore, we examined the HPV transcription profiles in esophageal (EC109 and EC9706), tongue (Tca83) and cervical (HeLa) cancer cell lines. Relative abundance of HPV transcripts was presented in parts per million (ppm). Overall, all these cell lines expressed E6, spliced E6 (E6*), E7, E1 and L1 transcripts (Fig. 1a). However, we noted that E1 transcripts were partially expressed in both EC109 and EC9706. Other HPV transcripts (E2, E4, E5, E8 and L2) were not detected in all the cell lines. These HPV genome profiling results were consistent with previous reports [36, 37].

Although relative abundance of transcripts originating from actively expressed regions of the viral genome were similar among these cell lines, differences in E6 and E7 transcript levels among cells were noted based on FPKM values. HeLa cells exhibited the highest level of HPV18 E6 transcripts $(115,690)$, followed by Tca83 $(98,246)$, EC9706 $(71,897)$ and EC109 $(70,874)$ (Table 1). Whilst spliced E6 variant I (E6"I) and E7 were markedly higher in EC109, EC9706 and HeLa (E6*I: 412,299 - 491,899; E7: 599,610 - 626,397) compared to Tca83 (E6"I: 293, 362; E7: 457,654) (Table 1). Furthermore, the E7 to E6 ratios in EC109 and EC9706 were nearly doubled relative to those in HeLa and Tca83 (Fig. 1b), whereas HeLa and Tca83 showed nearly doubled E6:E6*I ratios compared to EC109 and EC9706. Overall, these data revealed that while HPV18 genomes exhibited similar expression profiles in the esophageal, tongue and cervical cell lines examined, subtle differences in E6 and E7 expression patterns were noted.

HPV18 E6 in EC109, EC9706 and Tca83 targets p53 and its downstream targets in a similar manner

Following the differential expression of HPV18 oncoproteins reported above, we next examined whether E6 and E7 oncoproteins in esophageal (EC109 and EC9706) and tongue (Tca83) cancer cells target key cellular proteins in a similar manner to cervical cancer cells, such as HeLa. The cells were transfected with siRNA against HPV18 E6 and E7 (si18E6/E7). After $72 \mathrm{~h}$, total protein was extracted and the levels of proteins targeted by E6, including p53, p21 and hDlg, were analyzed via Western blotting.

We found that HPV18 E6 in all tested cell lines behaved similarly in perturbing its major target, p53, but not PDZ protein. We found that, like HeLa [Fig. 2a and b (i)], downregulation of HPV18 E6 in all the esophageal (EC109 and EC9706) and tongue (Tca83) SCC cell lines resulted in a significant rescue of p53 as well as its downstream transactivation target, p21 [Fig. 2a and b (ii, iii and iv)]. In addition, we observed increased levels of hDlg (a PDZ protein), in HeLa cells [Fig. 2a and b (i)] upon depletion of E6, but not in the esophageal and tongue SCC cell lines examined [Fig. 2a and b (ii, iii and iv)].

\section{pRB is not the major target of HPV E7 in EC109, EC9706 and Tca83}

As expected, we observed that downregulation of HPV18 E6 and E7 oncoproteins led to rescue of E7 targets (pRB, p130 and p107) in HeLa cells [Fig. 2a and b (ii, iii, iv)]. However, there was no significant change in the levels of pRB when E7 was downregulated in the esophageal (EC109 and EC9706) and tongue (Tca83) cell lines [Fig. $2 \mathrm{a}$ and $\mathrm{b}$ (ii, iii, iv)]. We observed significantly increased levels of p130 in both EC109 [Fig. 2a and b (ii)] and EC9706 [Fig. 2a and b (iii)], and increased p107 was only found in EC9706 [Fig. 2a and b (iii)]. Furthermore, downregulation of E7 in Tca83 did not affect the levels of $\mathrm{pRB}$ and its related pocket proteins [Fig. 2a and b (iv)].

\section{RB1, RB2 and p53 transcripts were not mutated in EC109, EC9706 and Tca83}

As we found that downregulation of HPV18 E6 and E7 had no effect on the E7 major target protein, pRB, in esophageal (EC109 and EC9706) and tongue (Tca83) cell lines, we further analyzed our RNA-seq data to look at FPKM values of RB1 (encoding for pRB), RB2 (encoding for p130) and TP53 (encoding for p53) transcripts in HeLa, EC109, EC9706 and Tca83. As shown in Table 1, expression of RB1, RB2 and TP53 in all these cell lines were comparable for all these HPV-positive cells. 
A.
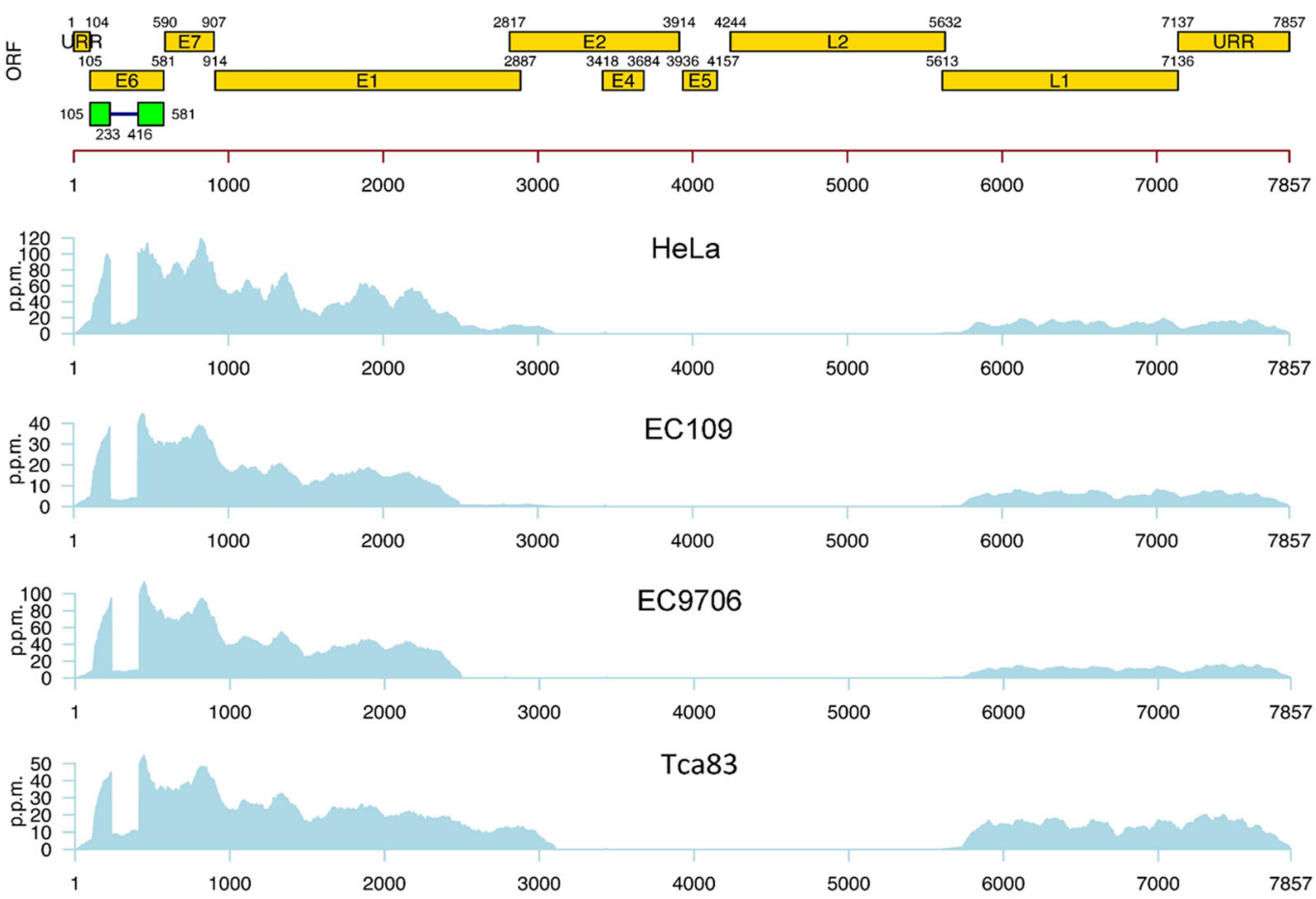

B. (i)

Ratio of E7 to E6

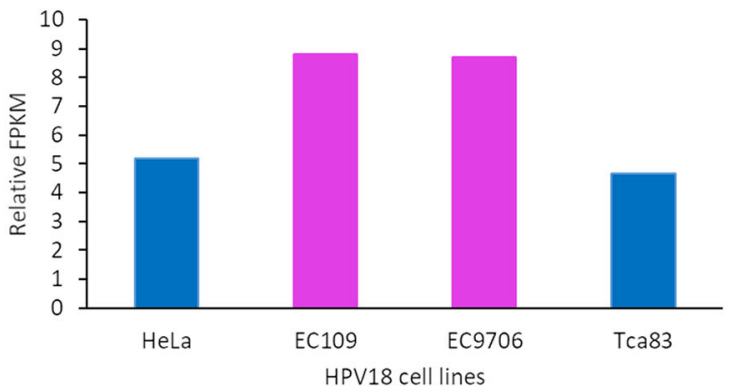

(ii)

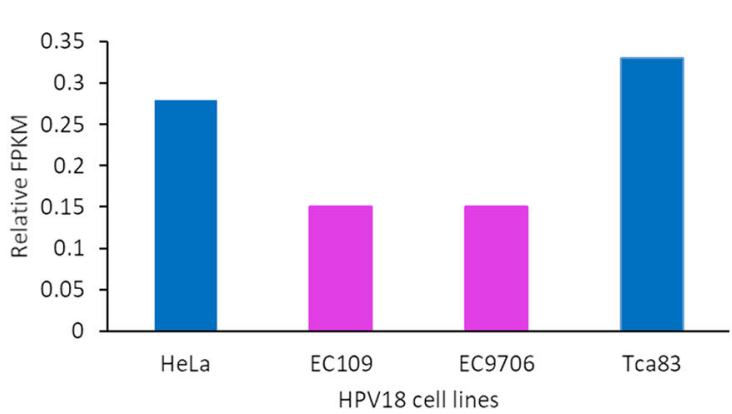

Fig. 1 Transcription profiles of the HPV18 genome in EC109, EC9706 and Tca83 cell lines measured through RNA sequencing. (a) Map of HPV genome showing protein-coding regions and their respective genomic locations. (b) Bar charts showing relative ratios of Fragments per kilobase of transcript per million reads mapped (FPKM) of (i) E7 to E6, and (ii) E6 to spliced E6 isoform I (E6 ${ }^{*}$ )

We further examined whether these transcripts harbored mutations that could potentially lead to amino acid alterations, and subsequently affect E7pRB recognition in EC109, EC9706 and Tca83 compared to HeLa. We observed that RB2 harbored same-sense mutations, corresponding to amino acid positions at T694, R679 and T864, while no exonic mutation was detected within RB1 (Additional file 1 ). On the other hand, we found all the cell lines carried the most common TP53 polymorphism converting Proline at amino acid codon 72 to Arginine (P72R) (Additional file 1), which is consistent with previous reports [38-40].
Tca83 cells, but not EC109 and EC9706, resemble HeLa cells in targeting ERK1/2 and MMP2 signaling pathways It is known that HPV18 oncoproteins can exert their oncogenic properties through targeting AKT [29], extracellular signal-regulated kinase (ERK) [30] and metalloprotease (MMP) [31, 32] pathways in cervical cancer cells, leading to cell survival, proliferation and metastasis. To date, the involvement of HPV18 oncoproteins in perturbing these pathways in esophageal and tongue SCC cell lines has not been clearly defined. This prompted us to look at the levels of AKT, ERK 1/2, MMP2 and MMP9 activities in esophageal (EC109 and EC9706) and tongue (Tca83) cells. In general, we 

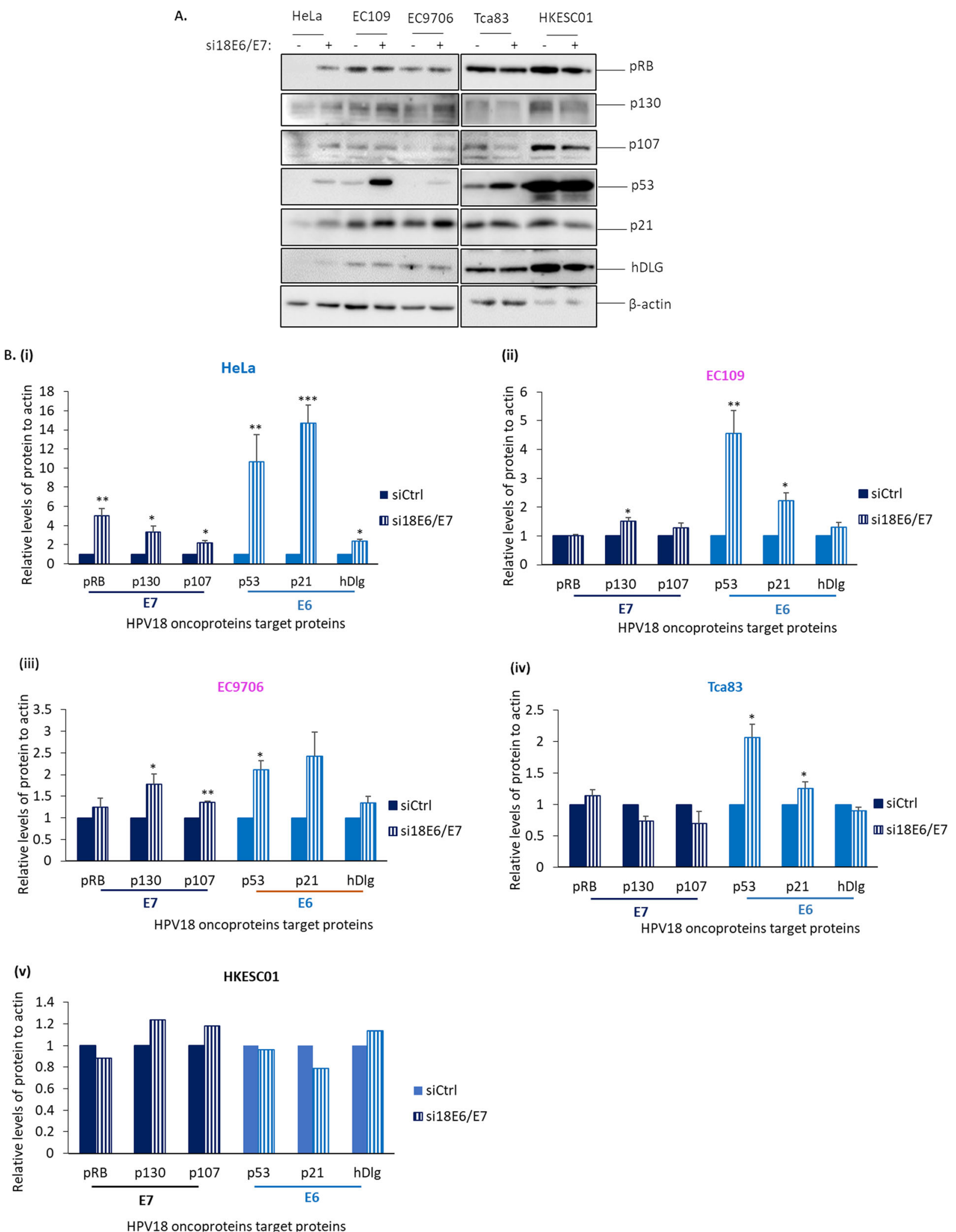

Fig. 2 (See legend on next page.) 
(See figure on previous page.)

Fig. 2 Effects of downregulation of HPV18 E6 and E7 on p53, pRB and its related pocket proteins in EC109, EC9706 and Tca83 cells. These cells were transfected with siRNA against control (-) or against HPV18 E6 and E7 (+). After 72 h, total lysate was collected and the levels of E6 and E7 target proteins were analyzed by Western blotting for the proteins as indicated. HeLa and HKESCO1 cells were included as positive and negative controls, respectively. a. Representative immunoblots showing levels of E7 (pRB, p103 and p107) (navy blue-colored bars) and E6 (p53, p21 and hDlg) (light blue-colored bars) target proteins. $\beta$-actin was included as a loading control. b. Bar graphs show quantitation of the levels of target proteins against control (siCtrl) in (i) HeLa, (ii) EC109, (iii) EC9706, (iv) Tca83 and (v) HKESC01 cells. Quantitation was performed using ImageJ software and statistical analysis was performed using Prism. Error bars represent mean \pm standard deviation (SD) $(n=4) .\left({ }^{*} P<0.05\right.$,

$\left.{ }^{* *} P<0.01,{ }^{* *} P<0.001\right)$

observed a higher basal level of both total and phosphorylated AKT at position S473 [pAKT(S473)], ERK 1/2 phosphorylated at position T202/Y204 [pERK1/2(T202/ Y204)], MMP2 and MMP9 in EC109, EC9706 and Tca83 compared to HeLa cells (Fig. 3a). Despite this, we found that Tca83 cells had similar behavior to HeLa cells in targeting ERK and MMP2 pathways, while both EC109 and EC9706 cells were distinct in targeting these pathways through HPV18 oncoproteins.

When HPV18 E6 and E7 in Tca83 cells were depleted using siRNA, we observed significant reduction in pERK1/2 (T202/Y204) and MMP2, together with a significant elevation in ERK1/2 in Tca83 [Fig. 3a, b (iii-v)]. These changes were also observed in HeLa cells. While MMP9 was markedly increased in HeLa cells, no significant change was observed in Tca83 cells [Fig. 3a, b (vi)].

Meanwhile, EC109 and EC9706 cells appeared to be different from HeLa cells. Downregulation of E6 and E7 resulted in a dramatic reduced level of AKT in EC109, but not in the other cells [Fig. 3a, Fig. 3b (i) and (ii)]. In addition, E6 and E7 downregulation had no significant effect on ERK activity, MMP2 and MMP9 levels in EC109 and EC9706. These results revealed that Tca83 had similar behavior to HeLa cells in regulating ERK1/2 activity and MMP2, and both esophageal SCCs were distinct from Tca83 and HeLa cells. Nevertheless, HPV18 oncoproteins appeared to perturb AKT activity in EC109 cells.

\section{Both Tca83 and HeLa cells require HPV18 oncoproteins to regulate the caspase pathway and proliferate}

HeLa cells are addicted to HPV oncoproteins to survive [41], partly through suppression of the caspase pathway $[42,43]$. We investigated whether this was reproducible in esophageal (EC109 and EC9706) and tongue (Tca83) cells using afore described siRNA approach to deplete E6 and E7.

We first looked at the levels of initiator (caspases 8 and 9) and effector (caspase 3) caspases. It has been shown that caspase 8 and 9 respond to extracellular apoptotic stimuli [44] and intracellular apoptosomes, respectively. This, in turn, leads to proteolytic and activation of effector caspases, including caspase 3 [45]. Our results showed that ablation of E6 and E7 in HeLa led to a significant increased levels of full length caspases 8, 9 and 3 [Fig. 4a, b (i), (iii) and (v)], as well as cleaved caspases 8 and 9 [Fig. 4a, b (iv) and (vi)]. Interestingly, we also observed increased levels of full length and cleaved caspases 8 and 9 in Tca83 upon depletion of HPV oncoproteins, indicating activation of caspases 8 and 9 [Fig. 4a, b (iii) to (vi)]. However, we did not observe activation of these initiator and effector caspases in EC109 and EC9706 (Fig. 4a and b). These results indicated that, like HeLa cells, E6 and E7 can suppress the caspase cascade in Tca83, but not in EC109 and EC9706.

We then wanted to know if esophageal and tongue SCC cells are dependent on HPV oncoproteins to proliferate. After E6 and E7 were depleted, we performed immunofluorescence assays to study the levels of Ki67 expression, a commonly used proliferation biomarker for cervical cancer. We also co-stained the cells with p53 as a measure of siRNA efficiency against HPV18 E6 and E7. Consistently, we observed a significant increased level of p53 upon depletion of HPV18 E6 and E7 in all HPV18-positive cells [Fig. 5a and b (ii)]. We observed a significant reduction in Ki67 expression in HeLa, EC9706 and Tca83, but not in EC109 upon ablation of E6 and E7 [Fig. 5a and b (i) and (ii)]. It is worth noting that Ki67 expression was relatively lower in HKESC01 than in other HPV-positive cells. Our results indicated that E6 and E7 promote proliferation of EC9706 and Tca83. Surprisingly, ablation of E6 and E7 was not adequate to initiate activation of caspase pathway in both EC109 and EC9706, as well as did not affect proliferation of EC109.

Overall, our data showed that, alike HeLa cells, Tca83 cells depend on HPV oncoproteins to attenuate initiator caspases and proliferate. In contrast, EC109 and EC9706 cells did not depend on HPV18 E6 and E7 to stimulate apoptosis. However, EC9706 cells require HPV oncoproteins to proliferate.

\section{Discussion}

The fact that high-risk HPV infection is associated with cancers of the uterine cervix, oropharynx, anus, vulvar and penis has been proven beyond doubt. However, the etiological role of HPV in cancers arise from esophageal and tongue remains controversial [15-18]. In this study, 


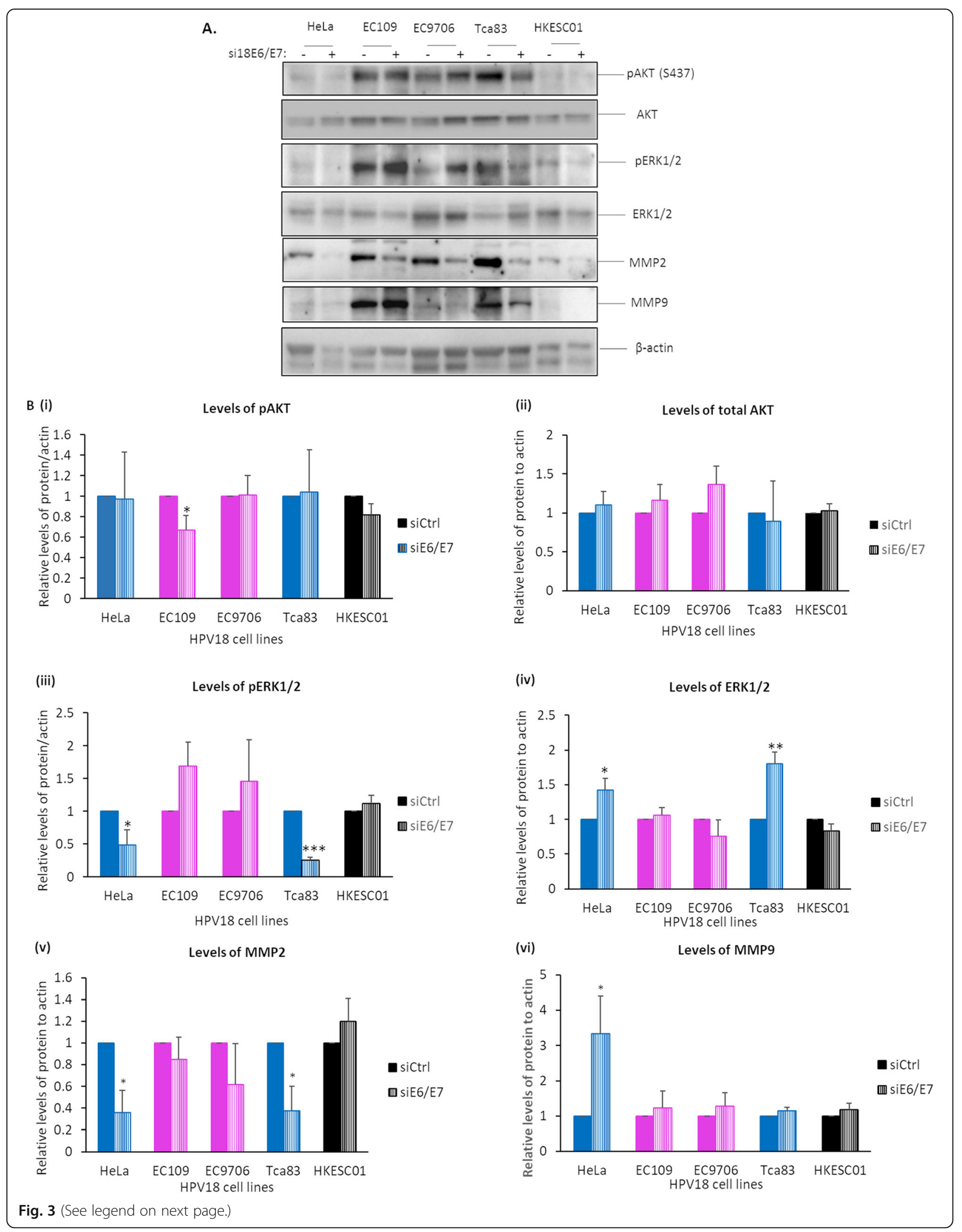


(See figure on previous page.)

Fig. 3 Effects of downregulation of HPV18 E6 and E7 on AKT, ERK1/2, MMP2 and MMP9 activity in EC109, EC9706 and Tca83 cells. These cells were transfected with siRNA against control (-) or against HPV18 E6 and E7 (+). After $72 \mathrm{~h}$, total lysate was collected and the levels of E6 and E7 target proteins were analyzed by Western blotting for the proteins as indicated. HeLa and HKESCO1 cells were included as positive and negative controls, respectively. a. Representative immunoblots showing levels of AKT phosphorylated at S437 [pAKT(S437)], pan-AKT, ERK1/2 phosphorylated at T202 and Y204 [pERK1/2(T202/Y204)], MMP2 and MMP9. $\beta$-actin was included as a loading control. b. Bar graphs show quantitation of the levels of target proteins against control in (i) HeLa (blue-colored bars), (ii) EC109 (magenta-colored bars), (iii) EC9706

(magenta-colored bars), (iv) Tca83 (blue-colored bars) and (v) HKESC01 (black-colored bars) cells. Quantitation was performed using ImageJ software and statistical analysis was performed using Prism. Error bars represent mean \pm standard deviation (SD) $(n=4)$. $\left(^{*} P<0.05\right.$,

$\left.{ }^{*} P<0.01,{ }^{* * *} P<0.001\right)$

we provided for the first time, a comparative molecular analysis among SCC cell lines originated from esophagus, tongue and uterine cervix to delineate their similarities and differences in terms of E6 and E7 transcript expressions and cellular targets.

Since viral integration with loss of E2 transcript is a hallmark of HPV-mediated oncogenesis, we first examined HPV transcription profiles in these cell lines. Our RNA-seq data showed partial expression of HPV genome in esophageal cell lines (EC109 and EC9706), which supports previous observations that integration of HPV genome has occurred [46]. Furthermore, we also observed a similar expression profile in the tongue cell line (Tca83), which suggests that viral integration has occurred. Interestingly, while the expression profiles of HPV genomes were similar among the three types of cancers, the relative ratios of E6, E7 and spliced E6 isoform I (E6"I) were different. Both EC109 and EC9706 expressed relatively higher levels of E7 and E6 I, while HeLa and Tca83 expressed relatively higher levels of E6. This expression pattern might reflect a functional resemblance of Tca83 to HeLa, and EC109 to EC9706, however, this does not necessarily reflect their respective ability in targeting known cellular proteins.

When we downregulated HPV18 E6 and E7 using siRNA approach, we expected to observe a rescue of E6 and E7 commonly targeted proteins for degradation: p53, p21 and hDlg by E6; pRB and its related pocket proteins, p130 and p107 by E7. In the esophageal and tongue cancer cell lines, E6 appeared to play the expected classical role in downregulating p53 and its downstream target p21 in a similar manner. This is most likely owing to the presence of similar p53 variant containing Arginine residue at codon 72 in all the cell lines examined, which is a form preferably degraded by E6 [39]. However, E6 did not degrade hDlg in esophageal and tongue cancer cell lines. As we did not measure other protein targets, the effect on PDZ proteins like hScribble [24] and MAGIs [23] remains to be clarified.

E7 might target different pathways in esophageal and tongue cancer cells compared to cervical cancer. Classically, pRB appears to be a better E7 degradation target in relative to p130 and p107. Intriguingly, we found that p130 was preferentially targeted by E7 in esophageal cancer cell lines. However, this was not observed in the tongue cancer cell line. This could be partly related to its lower E7 transcript expression than that of HeLa, EC109 and EC9706.

HPV-positive cells require HPV oncoproteins to survive and proliferate, which can occur through perturbation of signaling pathways, including AKT [29], ERK [30], suppression of the caspase cascade [42, 43], and MMPs for migration [31, 32]. AKT is known to possess a pro-survival role [41, 47], while ERK1/2 appears to be a dual-faced protein: anti- or pro-apoptotic kinase [48]. Activation of ERK1/2 was found to either activate or dampen caspase 8 and 9 [49,50]. Its activation can also activate MMPs [51]. In this study, we found that, consistent with expression patterns of HPV transcripts, tongue cancer cells had similar behavior to cervical cancer cells. In our hand, HPV oncoproteins in these two cells preferentially target the ERK $1 / 2$ pathway. This might occur through inactivation of the ERK1/2 upstream protein, mitogen-activated protein/extracellular signal-regulated kinase (MEK), as a result of feedback regulation by ERK1/2 [52]. This might in turn lead to inactivation of initiator caspase 8 and 9 , increased proliferation and MMP2-mediated migration.

The role of HPV oncoproteins in EC109 and EC9706 in cellular targeting are indeed distinct from that in HeLa and Tca83. Although these cells share certain degrees of similarity, EC109 and EC9706 can differ from each other in term of HPV18 genome transcripts and subset of cellular proteins targeted by HPV oncoproteins. Both esophageal cancer cell lines do not depend on HPV18 oncoproteins to perturb ERK1/2, caspases and MMPs pathways. The higher basal levels of ERK1/2 and MMPs compared to HeLa cells are sufficient to drive carcinogenesis independent of HPV18 oncoproteins. As both these cells were isolated from different patients, on top of HPV infection, these cells might be exposed to different cofactors, such as environmental factors and genetic predisposition, causing cells to undergo multi-steps carcinogenesis differently. At the molecular level, we found in EC109 that HPV18 oncoproteins can upregulate AKT activity, as reported previously [29], a process essential for cells to resist caspase-dependent apoptosis, proliferate and migrate independent of MMPs. Although EC9706 cells appeared 


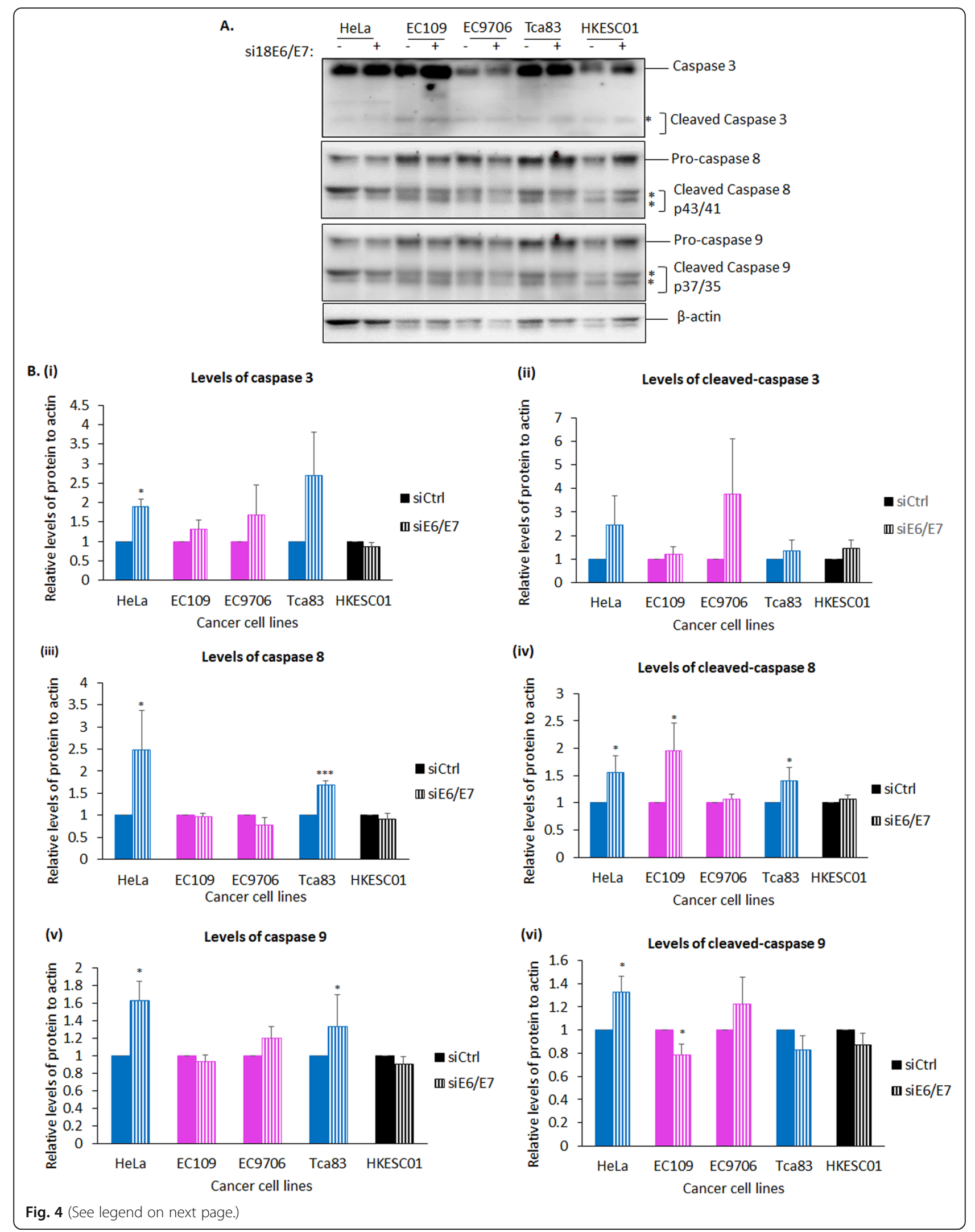


(See figure on previous page.)

Fig. 4 Effects of downregulation of HPV18 E6 and E7 on caspase-dependent apoptotic pathway in EC109, EC9706 and Tca83 cells. These cells were transfected with siRNA against control (-) or against HPV18 E6 and E7 (+). After $72 \mathrm{~h}$, total lysate was collected and the levels of E6 and E7 target proteins was analyzed by Western blotting for the proteins as indicated. HeLa and HKESCO1 cells were included as positive and negative controls, respectively. a. Representative immunoblots showing the levels of caspase 3, caspase 8, caspase 9 and their respective cleaved forms. $\beta$ actin was included as a loading control. b. Bar graphs show quantitation of the levels of the (i) caspase 3 and (ii) cleaved caspase 3, (iii) caspase 8 and (iv) cleaved caspase 8, (v) caspase 9 and (vi) cleaved 9, against control in HeLa (blue-colored bars), EC109 (magenta-colored bars), EC9706 (magenta-colored bars), Tca83 (blue-colored bars) and HKESC01 (black-colored bars). Quantitation was performed using ImageJ software and statistical analysis was performed using Prism. Error bars represent mean \pm standard deviation (SD) $(n=4) .\left({ }^{*} P<0.05,{ }^{* *} P<0.01,{ }^{* * *} P<0.001\right)$

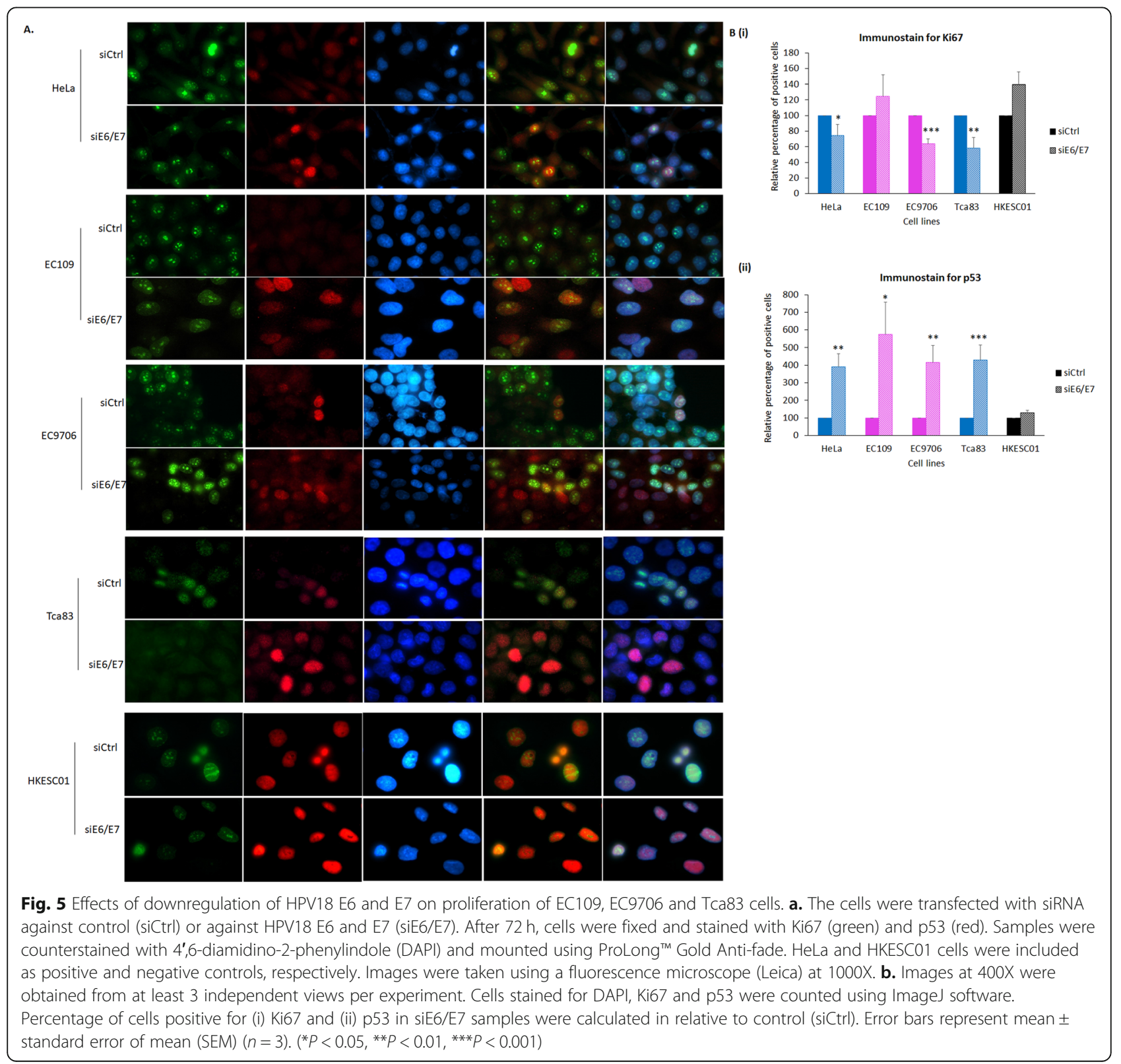


do not require HPV oncoproteins to manipulate signaling and apoptotic pathways examined in this study, they required HPV oncoproteins to proliferate. This could occur through perturbation of transforming growth factor- $\beta 1$ (TGF- $\beta 1$ ) signaling, which is important for epithelialmesenchymal transition (EMT) of EC9706 [53], and subsequently leading to inactivation of Fas-mediated apoptosis [54] in a caspase-independent fashion. However, this remains unknown and deserves further investigation.

Overall, in line with the model of HPV-mediated cervical carcinogenesis, p53 is the major target of E6 in the esophageal and tongue cancer cell lines examined, whilst p130 is preferentially targeted in esophageal cancer cells. In tongue cancer cells, ERK1/2 and MMP2 pathways appeared to be crucial, but not E7-pRB and AKT pathways. In esophageal cells, a high basal level of AKT, ERK1/2 and MMP activity compared to HPV-null esophageal and HPV-positive cervical cancer cells could be essential for multi-steps cancer progression. Nonetheless, our studies were bound to limitation. In our study, patientderived cell lines were used to elucidate the role of HPV18 oncoproteins in a monolayer culture system. Physiological relevant models, such as 3-dimensional (3D) culture and animal models should be employed to gain a better understanding on how HPV18 oncoproteins interact with tumour microenvironment and drive cancer progression.

\section{Conclusion}

In conclusion, our findings support that HPV could play an etiological role in esophageal and tongue cancers upon presence of other essential co-factors, such as environmental and genetic predisposition. Yet, the molecular pathways mediated by HPV oncoproteins could be different and are likely determined by tissue specific factors. Further studies focusing on esophageal and tongue cancers are needed to elucidate the full spectrum of HPV-associated cancers, and thus the potential benefits offered by HPV vaccines and treatment.

\footnotetext{
Abbreviations

E6*: Spliced E6; EC: Esophageal cancer; EMT: Epithelial-mesenchymal transition; ERK1/2: Extracellular signal-regulated kinase 1/2; FPKM: Fragments per kilobase per million reads mapped; HNC: Head and neck cancer; HPV: Human papillomavirus (HPV); MEK: Mitogen-activated protein/ extracellular signal-regulated kinase; MMPs: Metalloproteases; PAHs: Polycyclic aromatic hydrocarbons; PDZ: PSD95/Dlg/ZO-1; RB: Retinoblastoma; SCC: Squamous cell carcinoma; siRNA: Small interfering RNA
}

\section{Acknowledgements}

We are very grateful to Dr. JY Lim for valuable comments.

\section{Availability of data materials}

Available upon request from the corresponding author. The RNA sequence data is accessible through GEO Series accession number (SRA Accession: PRJNA530677)

\section{Consent to publish}

Not applicable.

\section{Authors' contributions}

PKSC, RZ and JL developed the study idea. SSB and ZC supervised experiments. KYCL and LYC executed experiments. PKSC and SSB interpreted the data. All authors involved in manuscript preparation. All authors read and approved the final manuscript.

\section{Funding}

No specific funding was received.

Ethics approval and consent to participate

Not applicable. This study did not use human or animal subject. Mammalian cell lines used in this study do not require ethics approval.

\section{Competing interests}

The authors declare no conflict of interest.

\section{Author details}

'Department of Microbiology, Faculty of Medicine, The Chinese University of Hong Kong, Shatin, Hong Kong. ${ }^{2}$ Beijing Key Laboratory of Environmental and Viral Oncology, College of Life Science and Bio-engineering, Beijing University of Technology, Beijing, China.

Received: 4 September 2019 Accepted: 29 November 2019

Published online: 12 December 2019

\section{References}

1. Ferlay J, Soerjomataram I, Dikshit R, Eser S, Mathers C, Rebelo M, Parkin DM, Forman D, Bray F. Cancer incidence and mortality worldwide: sources, methods and major patterns in GLOBOCAN 2012. Int J Cancer. 2015;136:E359-86.

2. Chaturvedi AK, Engels EA, Pfeiffer RM, Hernandez BY, Xiao W, Kim E, Jiang B, Goodman MT, Sibug-Saber M, Cozen W, Liu L, Lynch CF, et al. Human papillomavirus and rising oropharyngeal cancer incidence in the United States. J Clin Oncol. 2011;29:4294-301.

3. Mehanna H, Beech T, Nicholson T, El-Hariry I, McConkey C, Paleri V, Roberts S. Prevalence of human papillomavirus in oropharyngeal and nonoropharyngeal head and neck cancer-systematic review and metaanalysis of trends by time and region. Head Neck. 2013;35:747-55.

4. Abogunrin S, Di Tanna GL, Keeping S, Carroll S, Iheanacho I. Prevalence of human papillomavirus in head and neck cancers in European populations: a meta-analysis. BMC Cancer. 2014;14:968.

5. Abnet CC, Arnold M, Wei WQ. Epidemiology of esophageal squamous cell carcinoma. Gastroenterology. 2018;154:360-73.

6. Lin Y, Totsuka Y, He Y, Kikuchi S, Qiao Y, Ueda J, Wei W, Inoue M, Tanaka H. Epidemiology of esophageal cancer in Japan and China. J Epidemiol. 2013; 23:233-42.

7. Freedman ND, Abnet CC, Leitzmann MF, Mouw T, Subar AF, Hollenbeck AR, Schatzkin A. A prospective study of tobacco, alcohol, and the risk of esophageal and gastric cancer subtypes. Am J Epidemiol. 2007;165:1424-33.

8. Znaor A, Brennan P, Gajalakshmi V, Mathew A, Shanta V, Varghese C, Boffetta P. Independent and combined effects of tobacco smoking, chewing and alcohol drinking on the risk of oral, pharyngeal and esophageal cancers in Indian men. Int J Cancer. 2003;105:681-6.

9. Domper Arnal MJ, Ferrández Arenas Á, Lanas AÁ. Esophageal cancer: risk factors, screening and endoscopic treatment in Western and eastern countries. World J Gastroenterol. 2015;21:7933-43.

10. Deziel NC, Wei WQ, Abnet CC, Qiao YL, Sunderland D, Ren JS, Schantz MM, Zhang Y, Strickland PT, Abubaker S, Dawsey SM, Friesen MC, et al. A multiday environmental study of polycyclic aromatic hydrocarbon exposure in a high-risk region for esophageal cancer in China. J Expo Sci Environ Epidemiol. 2013;23:52-9.

11. De Stefani E, Deneo-Pellegrini H, Ronco AL, Boffetta P, Correa P, Aune D, Mendilaharsu M, Acosta G, Silva C, Landó G, Luaces ME. Meat consumption, cooking methods, mutagens, and risk of squamous cell carcinoma of the esophagus: a case-control study in Uruguay. Nutr Cancer. 2012;64:294-9.

12. Appah E, Ballard B, Izban M, Jolin C, Lammers P, Parrish D, Marshall D. A rapidly growing human papillomavirus-positive oral tongue squamous cell carcinoma in a 21-year old female: a case report. Oncol Lett. 2018;15:7702-6.

13. Haeggblom L, Attoff T, Yu J, Holzhauser S, Vlastos A, Mirzae L, ÄhrlundRichter A, Munck-Wikland E, Marklund L, Hammarstedt-Nordenvall L, Ye W, Ramqvist $\mathrm{T}$, et al. Changes in incidence and prevalence of human 
papillomavirus in tonsillar and base of tongue cancer during 2000-2016 in the Stockholm region and Sweden. Head Neck. 2018;41:1583-90.

14. Chung JJ, Okamoto Y, Coblitz B, Li M, Qiu Y, Shikano S. PI3K/Akt signallingmediated protein surface expression sensed by 14-3-3 interacting motif. FEBS J. 2009;276:5547-58.

15. Syrjänen K. Geographic origin is a significant determinant of human papillomavirus prevalence in oesophageal squamous cell carcinoma: systematic review and meta-analysis. Scand J Infect Dis. 2013;45:1-18.

16. Bognár L, Hegedűs I, Bellyei S, Pozsgai É, Zoltán L, Gombos K, Horváth ÖP, Vereczkei A, Papp A. Prognostic role of HPV infection in esophageal squamous cell carcinoma. Infect Agent Cancer. 2018;13:38.

17. Teng H, Li X, Liu X, Wu J, Zhang J. The absence of human papillomavirus in esophageal squamous cell carcinoma in East China. Int J Clin Exp Pathol. 2014;7:4184-93.

18. Koshiol J, Wei WQ, Kreimer AR, Chen W, Gravitt P, Ren JS, Abnet CC, Wang JB, Kamangar F, Lin DM, von Knebel-Doeberitz M, Zhang Y, et al. No role for human papillomavirus in esophageal squamous cell carcinoma in China. Int J Cancer. 2010;127:93-100.

19. Werness BA, Levine AJ, Howley PM. Association of human papillomavirus types 16 and 18 E6 proteins with p53. Science 80 1990;248:76-9.

20. Huibregtse JM, Scheffner M, Howley PM. A cellular protein mediates association of p53 with the E6 oncoprotein of human papillomavirus types 16 or 18. EMBO J. 1991;10:4129-35.

21. Scheffner M, Münger K, Byrne JC, Howley PM. The state of the p53 and retinoblastoma genes in human cervical carcinoma cell lines. Proc Natl Acad Sci U S A. 1991;88:5523-7.

22. Rho J, Lee $\mathrm{S}$, de Villiers EM, Choe J. Identification of cis-regulatory elements in the upstream regulatory region of human papillomavirus type 59. Virus Res. 1997;47:155-66.

23. Glaunsinger BA, Lee SS, Thomas M, Banks L, Javier R. Interactions of the PDZ-protein MAGI-1 with adenovirus E4-ORF1 and high-risk papillomavirus E6 oncoproteins. Oncogene. 2000;19:5270-80.

24. Nakagawa S, Huibregtse JM. Human scribble (Vartul) is targeted for ubiquitin-mediated degradation by the high-risk papillomavirus E6 proteins and the E6AP ubiquitin-protein ligase. Mol Cell Biol. 2000;20:8244-53.

25. Lee JO, Russo AA, Pavletich NP. Structure of the retinoblastoma tumour-suppressor pocket domain bound to a peptide from HPV E7. Nature. 1998;391:859-65.

26. Gonzalez SL, Stremlau M, He X, Basile JR, Münger K. Degradation of the retinoblastoma tumor suppressor by the human papillomavirus type 16 E7 oncoprotein is important for functional inactivation and is separable from proteasomal degradation of E7. J Virol. 2001;75:7583-91.

27. Helt AM, Galloway DA. Destabilization of the retinoblastoma tumor suppressor by human papillomavirus type 16 E7 is not sufficient to overcome cell cycle arrest in human keratinocytes. J Virol. 2001;75:6737-47.

28. Dyson N, Guida P, Münger K, Harlow E. Homologous sequences in adenovirus E1A and human papillomavirus E7 proteins mediate interaction with the same set of cellular proteins. J Virol. 1992;66:6893-902.

29. Menges CW, Baglia LA, Lapoint R, McCance DJ. Human papillomavirus type 16 E7 up-regulates AKT activity through the retinoblastoma protein. Cancer Res. 2006;66:5555-9.

30. Yuan H, Ito S, Senga T, Hyodo T, Kiyono T, Kikkawa F, Hamaguchi M. Human papillomavirus type 16 oncoprotein E7 suppresses cadherin-mediated cell adhesion via ERK and AP-1 signaling. Int J Oncol. 2009;35:309-14.

31. Zhu D, Ye M, Zhang W. E6/E7 oncoproteins of high risk HPV-16 upregulate MT1-MMP, MMP-2 and MMP-9 and promote the migration of cervical cancer cells. Int J Clin Exp Pathol. 2015;8:4981-9.

32. Shiau MY, Fan LC, Yang SC, Tsao CH, Lee H, Cheng YW, Lai LC, Chang YH. Human papillomavirus up-regulates MMP-2 and MMP-9 expression and activity by inducing interleukin-8 in lung adenocarcinomas. PLoS One. 2013;8:e54423.

33. Pertea M, Kim D, Pertea GM, Leek JT, Salzberg SL. Transcript-level expression analysis of RNA-seq experiments with HISAT, StringTie and Ballgown. Nat Protoc. 2016;11:1650-67.

34. Wang X, Meyers C, Wang HK, Chow LT, Zheng ZM. Construction of a full transcription map of human papillomavirus type 18 during productive viral infection. J Virol. 2011:85:8080-92

35. Massimi P, Narayan N, Thomas M, Gammoh N, Strand S, Strand D, Banks L. Regulation of the hDlg/hScrib/Hugl-1 tumour suppressor complex. Exp Cell Res. 2008;314:3306-17.

36. Groves IJ, Coleman N. Human papillomavirus genome integration in squamous carcinogenesis: what have next-generation sequencing studies taught us? J Pathol. 2018;245:9-18.
37. Nkili-Meyong AA, Moussavou-Boundzanga P, Labouba I, Koumakpayi $\mathrm{H}_{\text {, }}$ Jeannot E, Descorps-Declère S, Sastre-Garau X, Leroy EM, Belembaogo E, Berthet N. Genome-wide profiling of human papillomavirus DNA integration in liquid-based cytology specimens from a Gabonese female population using HPV capture technology. Sci Rep. 2019;9:1504.

38. Kawaguchi H, Ohno S, Araki K, Miyazaki M, Saeki H, Watanabe M, Tanaka S, Sugimachi K. p53 polymorphism in human papillomavirus-associated esophageal cancer. Cancer Res. 2000;60:2753-5.

39. Storey A, Thomas M, Kalita A, Harwood C, Gardiol D, Mantovani F, Breuer J, Leigh IM, Matlashewski G, Banks L. Role of a p53 polymorphism in the development of human papillomavirus-associated cancer. Nature. 1998;393: 229-34.

40. Network CGA. Comprehensive genomic characterization of head and neck squamous cell carcinomas. Nature. 2015;517:576-82.

41. DeFilippis RA, Goodwin EC, Wu L, DiMaio D. Endogenous human papillomavirus E6 and E7 proteins differentially regulate proliferation, senescence, and apoptosis in HeLa cervical carcinoma cells. J Virol. 2003;77: 1551-63.

42. Manzo-Merino J, Massimi P, Lizano M, Banks L. The human papillomavirus (HPV) E6 oncoproteins promotes nuclear localization of active caspase 8. Virology. 2014;450-451:146-52.

43. Du J, Chen GG, Vlantis AC, Chan PK, Tsang RK, van Hasselt CA. Resistance to apoptosis of HPV 16-infected laryngeal cancer cells is associated with decreased Bak and increased BCl-2 expression. Cancer Lett. 2004;205:81-8.

44. Tummers B, Green DR. Caspase-8: regulating life and death. Immunol Rev. 2017;277:76-89.

45. Kuida K. Caspase-9. Int J Biochem Cell Biol. 2000;32:121-4.

46. Liu CY, Li F, Zeng Y, zhong TM, Huang Y, Li JT, Zhong RG. Infection and integration of high-risk human papillomavirus in HPV-associated cancer cells Med Oncol. 2015;32:109.

47. Scheid MP, Woodgett JR. PKB/AKT: functional insights from genetic models. Nat Rev Mol Cell Biol. 2001;2:760-8

48. Lu Z, Xu S. ERK1/2 MAP kinases in cell survival and apoptosis. IUBMB Life. 2006:58:621-31.

49. Tran SE, Holmstrom TH, Ahonen M, Kahari VM, Eriksson JE. MAPK/ERK overrides the apoptotic signaling from Fas, TNF, and TRAIL receptors. J Biol Chem. 2001:276:16484-90.

50. Bin PG, Choi Y, Kim YS, Lee HK, Kim D, Hur DY. ROS and ERK1/2-mediated caspase- 9 activation increases XAF1 expression in dexamethasone-induced apoptosis of EBV-transformed B cells. Int J Oncol. 2013;43:29-38.

51. Moulik S, Pal S, Biswas J, Chatterjee A. Role of ERK in modulating MMP 2 and MMP 9 with respect to tumour invasiveness in human cancer cell line MCF-7 and MDA-MB-231. J Tumor. 2014;2:87-98.

52. Hong SK, Wu PK, Karkhanis M, Park JI. ERK1/2 can feedback-regulate cellular MEK1/2 levels. Cell Signal. 2015;27:1939-48.

53. Xuan X, Zeng Q, Li Y, Gao Y, Wang F, Zhang H, Wang Z, He H, Li S. Aktmediated transforming growth factor- $\beta 1$-induced epithelial-mesenchymal transition in cultured human esophageal squamous cancer cells. Cancer Gene Ther. 2014;21:238-45

54. Jung YJ, Kim JY, Park JH. TGF- $\beta 1$ inhibits Fas-mediated apoptosis by regulating surface Fas and CFLIPL expression in human leukaemia/ lymphoma cells. Int J Mol Med. 2004;13:99-104.

\section{Publisher's Note}

Springer Nature remains neutral with regard to jurisdictional claims in published maps and institutional affiliations.

Ready to submit your research? Choose BMC and benefit from:

- fast, convenient online submission

- thorough peer review by experienced researchers in your field

- rapid publication on acceptance

- support for research data, including large and complex data types

- gold Open Access which fosters wider collaboration and increased citations

- maximum visibility for your research: over $100 \mathrm{M}$ website views per year

At $\mathrm{BMC}$, research is always in progress.

Learn more biomedcentral.com/submission 